\title{
Review of palivizumab in the prophylaxis of respiratory syncytial virus (RSV) in high-risk infants
}

\author{
Asunción Mejías \\ Octavio Ramilo \\ Division of Pediatric Infectious \\ Diseases, The University of Texas \\ Southwestern Medical Center and \\ Children's Medical Center, Dallas, \\ Texas, USA
}

\begin{abstract}
In respiratory syncytial virus (RSV) disease the balance between the innate and adaptive immune responses determines the expression of the pathological phenotype favoring the development of acute bronchiolitis, and in certain children the development of recurrent wheezing. While humoral antibody plays a major role in protection against disease, T-cell immunity targeted to viral proteins appears to terminate viral infection. At the moment, treatment modalities for acute RSV infection do not effectively modify the course of the disease, and RSV vaccine development has shown conflicting results. To date, however, passive immunoprophylaxis with monoclonal antibodies is the only strategy that has demonstrated consistent efficacy in reducing RSV hospitalizations in high-risk children. The potential benefit of new strategies for prevention and treatment of RSV infections should be evaluated with respect to both the acute infection as well as the chronic respiratory manifestations induced by RSV.
\end{abstract}

Keywords: respiratory syncytial virus, palivizumab, infants

\section{The virus}

Respiratory syncytial virus (RSV) is a member of the Pneumovirus genus, Paramyxovidae family. Its negative-sense single-stranded RNA genome contains 10 genes that encode 11 proteins. Five of these proteins are involved in RNA synthesis: the nucleocapside $\mathrm{N}$ protein, the $\mathrm{L}$ protein (polymerase), the phosphoprotein $\mathrm{P}$, and the M2-1 and M2-2 proteins (Collins and Crowe 2007). Four other RSV proteins are associated with the lipid bilayer to form the viral envelope: the matrix M protein, two glycosylated surface proteins, the $\mathrm{F}$ and $\mathrm{G}$ proteins, and a small hydrophobic SH protein. The $F$ and $G$ proteins are crucial for the infectivity and pathogenesis of the virus. The $G$ protein mediates attachment of the virus to respiratory epithelial cells, whereas the $\mathrm{F}$ (fusion) protein facilitates entry of the virus by fusing viral and cellular membranes and insertion of the RNA into the host cell inducing the formation of the characteristic syncytia (Hall 2000). The F and G proteins carry the antigenic determinants that elicit the production of neutralizing antibodies by the host. The remaining two proteins, NS1 and NS2, are nonstructural accessory proteins involved in modulating the host response to infection. NS1 and NS2 are abundantly present in infected cells and confer virus resistance to type-I interferons enhancing viral replication and survival (Spann et al 2004). Compared with other close relatives, in RSV the M2-1 protein is essential for the viability of the virus and in its absence transcription terminates nonspecifically and results in expression of NS1 and NS2 alone (Fearns and Collins 1999).

RSV displays minimal antigenic heterogeneity; however, there are two major RSV subtypes, A and B, which cocirculate each year. Much of the antigenic diversity between and within RSV subtypes is due to variations in the G glycoprotein, with as little as 35\% homology between G glycoprotein of strains A and B (Johnson et al 2004). 
It is not clear whether one subtype is more virulent than the other (Devincenzo 2004; Chavez-Bueno et al 2005). Antibody responses to the $\mathrm{F}$ protein are often cross-reactive for RSV A and B, while antibody responses to the G protein are subtype specific.

\section{Epidemiology}

RSV is the leading viral pathogen responsible for lower respiratory tract infection (LRTI) requiring hospitalization in infants and young children worldwide. In US alone, it is estimated that at least 140,000 hospitalizations can be attributed to RSV LRTI each year (Shay et al 1999). The reservoir is exclusively human. RSV outbreaks occur annually and predictably, lasting from late fall through early spring in temperate climates but it can circulate all year long in areas close to the equator (Mejias et al 2005a).

The spectrum of RSV disease is wide, ranging from mild upper respiratory tract infection (URI), LRTI, to respiratory failure. Primary RSV infection is almost always symptomatic. Infection with RSV is one of the most common diseases of childhood. It is responsible for $50 \%-90 \%$ of children hospitalized for bronchiolitis and for 5\%-40\% due to pneumonia (Psarras et al 2004). Approximately two-thirds of infants are infected with RSV during the first year of life, and $90 \%$ have been infected one or more times by 2 years of age (Glezen et al 1986). Studies conducted in many different countries over the last 20 years have consistently demonstrated that premature infants and children with chronic lung disease (CLD) and congenital heart disease (CHD) are at greater risk for severe RSV infection. In addition, RSV has been associated with severe respiratory illness in the elderly, immunocompromised patients as well as in previously healthy adults (Hall et al 1978).

In addition to the acute morbidity, different studies have shown over the past 10 years that young children who had RSV LTRI are at increased risk for developing recurrent wheezing and possibly asthma later in childhood (Stein et al 1999; Sigurs et al 2005).

\section{RSV treatment}

While significant advances in the knowledge of RSV biology, immunology, and epidemiology have been made in the last 40 years, there continues to be significant controversy over the optimum management of infants with acute RSV infection.

It has been suggested that RSV viral loads in the respiratory tract correlates with disease severity (Malley et al 1998; Buckingham et al 2002). However, as the disease progresses it is mainly the immune response responsible for the clinical manifestations and the persistence of symptoms (Malley et al 1998; Jafri et al 2004). Since the initial isolation of the virus, there have been considerable efforts to develop effective therapies for RSV infection: some directed against the virus such as ribavirin, others to modulate the inflammatory response including steroids and anti-leukotrienes, and others to reduce the symptoms such as bronchodilators and heliox or nebulized hypertonic saline (Kuzik et al 2007).

To date, however, there is no specific treatment that is uniformly used for the management of this infection in previously healthy infants (Red Book 2006). Close monitoring of the clinical evolution and supportive care of patients with intravenous fluids and oxygen remain the mainstay of treatment of RSV disease in the great majority of patients (Mejias et al 2005a).

\section{RSV prophylaxis}

The lack of effective therapy against RSV infection makes prophylactic interventions the best strategy to avoid the acute and chronic complications of the disease (Palivizumab 1998; Simoes et al 2007). The peak incidence of severe RSV disease is between 2 and 3 months of age. The main risk factors for severe RSV disease in children include prematurity, CLD and CHD. Additional risk factors include male gender, birth during the first half of the RSV season, younger age of RSV acquisition, and malnutrition (Parnes et al 2003). In addition there are several environmental factors that are associated with increased RSV disease severity such as crowding, passive exposure to tobacco smoke, day-care attendance, and the number of older school-age siblings (Chavez-Bueno et al 2006). Thus, education of parents and other caregivers about methods to decrease the infant's exposure to RSV and to other factors that may contribute to the severity of the infection must form the basis of any RSV prophylaxis program (Red Book 2006). Because RSV is transmitted by direct or close contact with contaminated secretions and the virus is known to persist for hours on environmental surfaces and for 30 minutes or longer on hands, emphasis on hand hygiene is crucial (Red Book 2006).

Infants affected with RSV shed large quantities of virus in their nasopharyngeal secretions and saliva for up to 3 weeks after the acute episode of RSV LRTI (Hall et al 1976). High-risk infants and children should not be exposed to individuals with respiratory infections and if at all possible, they should not be in settings (eg, child care centers) where such exposures are likely. Exposure to tobacco smoke must be eliminated since it has been associated with more severe RSV disease. These measures will have the added benefit of decreasing transmission of other respiratory pathogens. 
In addition to these hygienic measures, passive immunoprophylaxis of high-risk children initially with a polyclonal RSV Immune Globulin Intravenous (RSV-IGIV; RespiGam $^{\mathrm{TM}}$, MedImmune Inc, Gaithersburg, MD, USA) and then with a monoclonal antibody, palivizumab (MEDI-493; Synagis $^{\circledR}$, MedImmune Inc, Gaithersburg, MD, USA), has been shown to reduce hospitalizations due to RSV infections, the surrogate marker for severe RSV lower respiratory tract disease in clinical trials. Despite this success, it is important to remember that most infants with severe RSV disease requiring hospitalization are previously healthy, term infants with no identifiable risk factors.

\section{Passive immunization}

\section{Polyclonal antibodies}

RSV-IGIV is a hyperimmune polyclonal human intravenous antibody preparation that was prepared from multiple donors. It was licensed by the Food and Drug Administration (FDA) in 1996. Two multicenter, randomized clinical trials demonstrated that monthly RSV-IGIV infusions of $750 \mathrm{mg} / \mathrm{kg}$ in children with either prematurity ( $\leq 35$ weeks of gestation, $<6$ months of age) or BPD (age $\leq 24$ months) resulted in a $41 \%-63 \%$ reduction in RSV hospitalization (Groothius et al 1993; PREVENT 1997). An additional multicenter trial of RSV-IGIV in children $<48$ months of age with CHD showed a nonsignficant $31 \%$ decrease in RSV hospitalizations. A significant increase in cyanotic episodes and cardiac surgery-related deaths occurred among those children with cyanotic CHD and right to left shunt (Simoes et al 1998). Thus, RSV-IVIG should not be used in children with CHD. Other disadvantages of prophylaxis with RSV-IGIV include the need for intravenous access; the fluid load $(15 \mathrm{~mL} / \mathrm{kg})$ required to deliver the drug, the potential for transmission of blood-borne pathogens, and the interference with the antibody response to live-virus vaccines. On the other hand, since it is a polyclonal preparation it also offered protection against a variety of pathogens besides RSV. RespiGam, the initially licensed RSV-IVIG preparation, is no longer marketed in the US; its use has been replaced by palivizumab.

\section{Monoclonal antibodies}

Palivizumab

Palivizumab is a neutralizing humanized mouse IgG1 monoclonal antibody directed against one of the two most antigenically stable sites of the F glycoprotein of the virus, site A (Beeler et al 1989). It blocks the fusion of the virus to the host epithelial cell. Palivizumab was completely engineered in the laboratory, and as such $95 \%$ is of human origin and only $5 \%$ murine. Due to the more conserved nature of these epitopes within the $\mathrm{F}$ protein, palivizumab shows a high degree of cross-reactivity among RSV subtypes A and B and is about 50-fold more potent than polyclonal preparations (Johnson et al 1997). It was licensed by the FDA in 1998 (Palivizumab 1998) for prevention of severe RSV disease in preterm infants and children with CLD. More recently, palivizumab was also approved for use in children with hemodynamically significant CHD (Feltes et al 2003). The safety and efficacy of palivizumab in high-risk infants and children was demonstrated in 2 large randomized, placebo-controlled trials.

The IMpact Trial assessed the safety and efficacy of intramuscular palivizumab at a dose of $15 \mathrm{mg} / \mathrm{kg}$ provided every 30 days during the RSV season for prevention of RSV hospitalization (Palivizumab 1998). This international, randomized, double blind, placebo-controlled study enrolled $1,502$ children who were either were 1$) \leq 35$ weeks of gestation and $\leq 6$ months of age, or 2$) \leq 24$ months of age with CLD that required medical treatment (ie, supplemental oxygen, steroids, bronchodilators, or diuretics) within the previous 6 months at the start of the RSV season. Palivizumab prophylaxis resulted in a $55 \%$ overall decrease of RSV-related hospitalization compared to placebo-treated controls. Subgroup analyses by degree of prematurity, and by absence of CLD also yielded significant reductions in RSV hospitalization. Palivizumab prophylaxis also demonstrated clinical efficacy in several secondary endpoints: it reduced the length of hospitalization for RSV infection, decreased requirement for supplemental oxygen, decreased number of days of moderate or severe lower respiratory tract illness, and decreased requirement for hospitalization in the intensive care unit.

Data obtained from the Palivizumab Outcomes Registry, a prospective nationwide registry of 2,116 high-risk children who received palivizumab after its FDA licensure, demonstrated total RSV hospitalization rates of $2.9 \%$ compare to the $4.8 \%$ reported in the Impact-RSV trial. Thus, the postlicensure real world experience has shown even greater efficacy in reducing hospitalization rates in high-risk children. The majority of the RSV hospitalizations reported to the Registry occurred between the first and second doses, possibly reflecting lower trough concentrations of palivizumab following a single dose (Parnes et al 2003)

Palivizumab also has shown benefit in children with CHD. From 1998 to 2002 during 4 consecutive RSV seasons, an international, multicenter, randomized, double blind, placebocontrolled study was conducted. This trial evaluated the safety, tolerance and efficacy of palivizumab for prevention of RSV hospitalization in 1,287 children who were $\leq 24$ months of age 
with hemodynamically significant CHD (Feltes et al 2003). Eligible children were $\leq 24$ months old and were stratified at entry to a cyanotic or acyanotic group. This study demonstrated that palivizumab was safe in children with cardiac disease and that its use decreased by $45 \%$ the rate of RSV hospitalization. Although the trial was not powered to analyze differences among subgroups, hospitalizations were reduced by $29 \%$ in those with cyanotic CHD while among those children with acyanotic lesions there was a significant reduction of $58 \%$. In addition, among all study children, there was a $56 \%$ decrease in total days of RSV-associated hospitalization per 100 children and a $73 \%$ decrease in total RSV-associated hospital days with supplemental oxygen per 100 children. The percentage of children who died was similar in both the palivizumab (3.3\%) and placebo (3.7\%) groups. None of the fatalities were attributed to the study drug. Palivizumab serum concentrations decreased by $58 \%$ after cardiopulmonary bypass, and it is recommended that a postoperative dose of palivizumab be provided in those children who continue to require prophylaxis after such surgery.

The results of this study led to approval of palivizumab by the FDA for prophylaxis in this high-risk group of children. Similarly, the palivizumab Outcomes Registry prospectively collected data on 1,490 subjects with CHD who received RSV prophylaxis with palivizumab from 2000 to 2004. This study demonstrated that total RSV hospitalization rates in those subjects with either cyanotic or acyanotic CHD who received palivizumab were low and decreased from the first to the fourth season (4.3\% vs 1.5\% respectively) (Cohen et al 2008), confirming the efficacy of palivizumab in this setting.

Palivizumab has not been associated with an increased risk of localized or systemic adverse events. Specifically, there has been no significant difference in hematologic, renal, or hepatic abnormalities between palivizumab and placebo recipients, even with repeated dosing during a second season of prophylaxis. In the IMpact trial, fever, mild injection site erythema, induration or swelling occurred in $3 \%$ of recipients. Antibodies to palivizumab have been seen transiently among both drug and placebo recipients, but this has not been associated with adverse reactions. Following licensure of palivizumab, anaphylaxis after re-exposure and acute hypersensitivity reactions on initial and re-exposure to palivizumab have been reported rarely $(<1$ in 100,000$)$.

\section{Recommendations for palivizumab prophylaxis}

The American Academy of Pediatrics (AAP) has issued a policy statement for the use of palivizumab for prophylaxis of RSV infections in high-risk children (AAP 2003). Palivizumab is administered intramuscularly at a dose of $15 \mathrm{mg} / \mathrm{kg}$ monthly (every 30 days) during the RSV season, which typically in the US starts in November and ends in March or April. A maximum of 5 doses is generally sufficient prophylaxis during one season. This recommended dose and schedule assures the maintenance of serum palivizumab concentrations of $\geq 40 \mu \mathrm{g} / \mathrm{mL}$, a concentration that has shown to decrease pulmonary RSV replication in the cotton rat model by $>100$-fold (Johnson et al 2007). Different studies have shown that protective palivizumab serum through concentrations $(>40 \mu \mathrm{g} / \mathrm{mL})$ increased after the second dose of $15 \mathrm{mg} / \mathrm{kg} / \mathrm{month}$ (Saez-Llorens et al 1998). Thus, it is important to avoid delaying administration of the second injection dose in order to achieve adequate protective serum levels. High-risk infants and children should receive palivizumab before hospital discharge. Once a child qualifies for prophylaxis, administration should continue throughout the RSV season and not stop at the point he or she reaches any certain age. Since some children experience more than one RSV infection during a given season, infants and children who develop a RSV infection while receiving palivizumab should continue to receive prophylaxis after recovery from their acute infection. Palivizumab can be given with routine immunizations since it does not interfere with the immunologic response to vaccines. Palivizumab is now available in a preservative-free liquid formulation with either $50 \mathrm{mg}$ or $100 \mathrm{mg}$ of palivizumab (Synagis ${ }^{\circledR}$, MedImmune, Inc.) in $0.5 \mathrm{~mL}$ or $1 \mathrm{~mL}$ vials, respectively (Palivizumab 2005). The liquid form is stable at refrigerator temperatures. The lyophilized form continues to be available. Palivizumab can be given with routine childhood immunizations since it does not interfere with the immunologic response to vaccines. Although RSV mutants resistant to palivizumab have been derived in the laboratory (Zhao et al 2004a, b), no palivizumab-resistant mutants have been described in humans (Devincenzo et al 2004).

The recommendations issued by the AAP for the use of palivizumab in high-risk children are provided in Figure 1 (AAP 2003). For infants born between 32 weeks, 1 day and 35 weeks, 0 days of gestation, the AAP recommends that prophylaxis be considered only if 2 or more risk factors are present. Although palivizumab has been shown to decrease RSV hospitalization in these infants, and infants 33-35 weeks of gestation may have adverse hospital outcomes secondary to RSV (Beyer et al 2004), the health-care expenditures of prophylaxis makes routine administration of palivizumab problematic in this group of infants. 


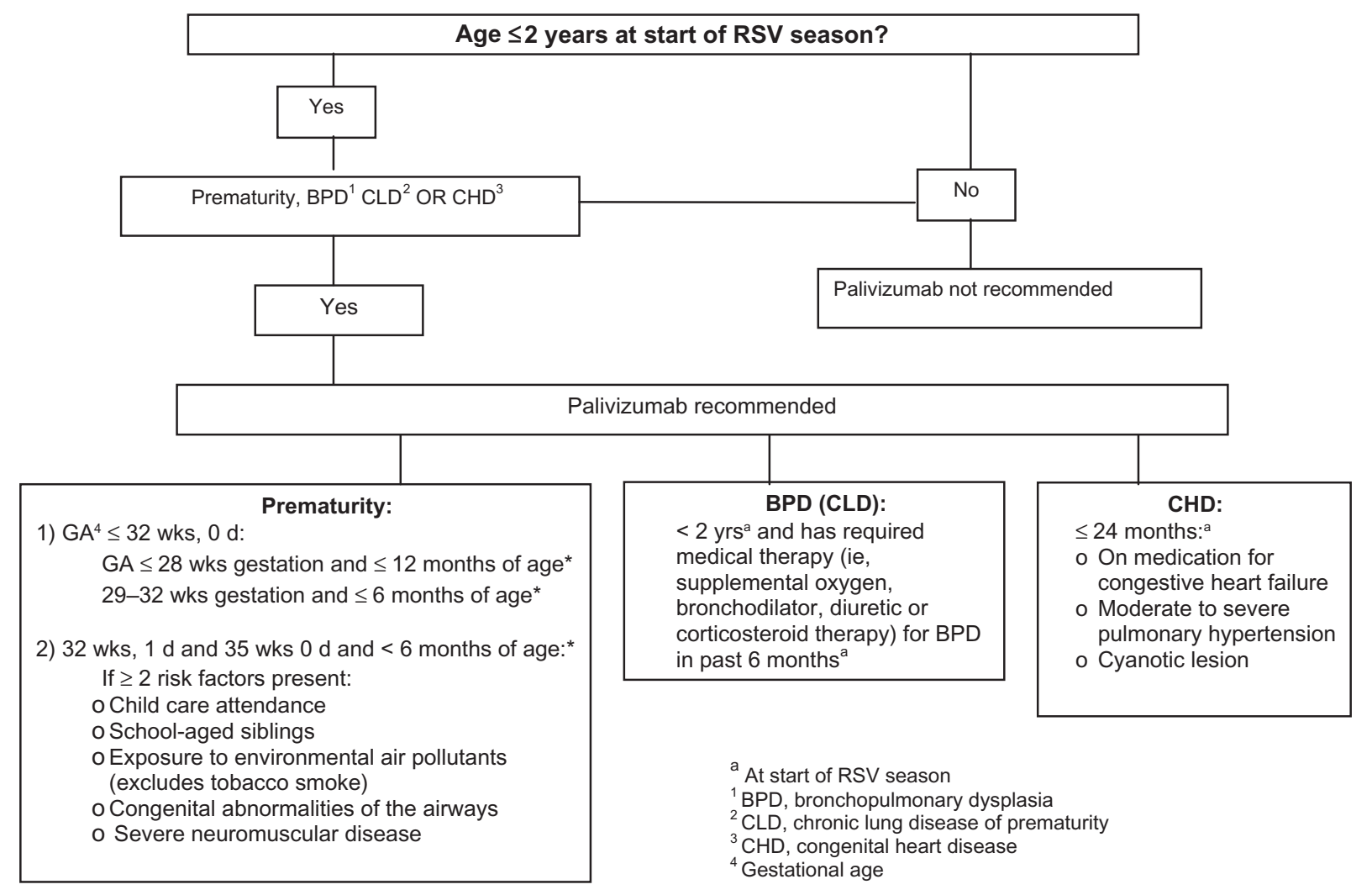

Figure I Recommendations for respiratory syncytial virus (RSV) prophylaxis in high-risk children.

Children with hemodynamically significant CHD are candidates for prophylaxis. However, the degree of physiologic cardiovascular compromise must be assessed. Children with the following cardiac lesions are not at increased risk for severe RSV infection and generally are not considered candidates for immunoprophylaxis: 1) hemodynamically insignificant CHD such as secundum atrial septal defect, small ventricular septal defect, pulmonic stenosis, uncomplicated aortic stenosis, mild coarctation of the aorta, and patent ductus arteriosus; 2) lesions adequately corrected by surgery unless there is continued requirement of medication for congestive heart failure; and 3) mild cardiomyopathy not requiring medical therapy.

\section{Motavizumab}

Monoclonal antibody variants derived from palivizumab have been generated by modifying antibody binding kinetics that resulted in increased binding avidity and viral neutralization activity (Mejias et al 2004b). One of these antibodies, motavizumab (Medi-524 or Numax ${ }^{\mathrm{TM}}$ ), a second-generation $\mathrm{mAb}$, has been shown to decrease lower and upper airway RSV loads in cotton rats by 50 - to 110 -fold compared with palivizumab.
A recent multinational, randomized double blind, phase III clinical trial assessed the safety and relative efficacy of motavizumab and palivizumab at reducing RSV specific hospitalizations and outpatient medically attended lower respiratory tract infections (LRIs). Over 2 consecutive RSV seasons 6,635 pre-term infants $\leq 35$ weeks gestation and $\leq 6$ months of age or preterm infants $\leq 24$ months of age with CLD requiring treatment were randomized to receive palivizumab $(\mathrm{n}=3,3326)$ or motavizumab $(\mathrm{n}=3,329)$ at $15 \mathrm{mg} / \mathrm{kg}$ IM monthly, and followed for 150 days. Administration of either $\mathrm{mAb}$ resulted in very low rates of RSV hospitalization ( $1.4 \%$ vs $1.9 \%$ for motavizumab vs palivizumab respectively). However, motavizumab showed better protection against RSV outpatient medically attended LRIs with a 50\% relative reduction in RSV specific LRIs compared with palivizumab ( $2 \%$ vs $3.9 \%$, respectively $(\mathrm{p}<0.01)$ (Carbonell-Estrany et al 2007). A phase III clinical study is ongoing to evaluate safety and efficacy of motavizumab versus palivizumab at reducing severe RSV disease in patients with hemodinamically significant CHD. Most recently, a third-generation mAb, Numax-YTE, has been generated with the intent to extend the serum half-life of the mAbs in humans. If successfully developed, this drug may offer the 
opportunity for less frequent dosing, obviating the need for the monthly treatments that are required with palivizumab (Mejias et al 2004a).

\section{Prophylaxis in other high-risk populations}

There are other several high-risk groups that may benefit from prophylaxis; however insufficient data exist to recommend its routine use. In these situations physicians caring for these patients make individualized decisions on the basis of the available data and the unique situation of each patient.

a) Viral respiratory infections, including RSV infections, are commonly associated with acute pulmonary exacerbations in patients with cystic fibrosis (CF) (Wang et al 1984). The short-term morbidity of RSV infection in young children with CF (length of hospitalization and ICU stay) appears to be comparable to that of children with CLD (Arnold et al 1999). A recent retrospective cohort study in 75 children diagnosed with $\mathrm{CF}$ at $\leq 18$ months of age (35 of whom received palivizumab prophylaxis) showed a reduced but not significant length of hospitalization during the first RSV season in patients that received the prophylaxis, suggesting that young children with CF may benefit from the use of palivizumab (Giebels et al 2008). Results are pending of a phase IV multicenter, randomized, double blind, placebo-controlled study on the safety and tolerance of palivizumab in children $\leq 24$ months of age with cystic fibrosis.

b) RSV can cause serious respiratory illness among solid organ transplant (SOT) patients, yet there is no standard recommendation for RSV immunoprophylaxis. To learn the current practices for the prevention of RSV disease in pediatric SOT candidates and recipients in the US, a 24-questionnaire survey was mailed to 108 SOT programs in 2006. The types of SOT programs included: liver, heart, lung, intestine, and heart-lung types of transplants. Among the programs that completed the survey, 49\% reported the use of prophylaxis with palivizumab with similar rates among recipients of all organ types. No data on RSV hospitalizations and outcomes were obtained and this information is crucial to develop a more rational approach to RSV immunoprophylaxis in these high-risk patients (Fonseca-Aten et al 2007).

\section{Potential long-term benefits of anti-RSV prophylaxis}

In addition to reducing the acute morbidity, there also may be potential benefits from prevention of long term RSV sequelae such as recurrent wheezing and asthma. In an animal model of RSV infection, prophylactic administration of both palivizumab and motavizumab significantly reduced the development of airway hyperreactivity and chronic airway inflammation (Mejias et al 2004b, 2005b). In humans, a recent double cohort multicenter study showed that preterm infants who received palivizumab prophylaxis during their first RSV season had a significant lower risk of subsequent recurrent wheezing during a 24-month follow-up compared with age-matched controls who did not receive prophylaxis (Simoes et al 2007). Despite its limitations this is a crucial study that demonstrates for the first time in humans that interventions directed against acute RSV can provide a long-term benefit. Future, prospective, randomized, placebocontrolled studies should be developed to confirm these important findings.

\section{Disclosures}

Drs Mejías and Ramilo belong to the MedImmune speaker's bureau. Dr Ramilo has also received research grants from MedImmune and Abbott.

\section{References}

[AAP] American Academy of Pediatrics Committee on Infectious Diseases and Committee on Fetus and Newborn. 2003. Revised indications for the use of palivizumab and respiratory syncytial virus immune globulin intravenous for the prevention of respiratory syncytial virus infections. Pediatrics, 112:1442-6.

Arnold SR, Wang EE, Law BJ, et al. 1999. Variable morbidity of respiratory syncytial virus infection in patients with underlying lung disease: a review of the PICNIC RSV database. Pediatric Investigators Collaborative Network on Infections in Canada. Pediatr Infect Dis J, 18:866-9.

Beeler JA, van Wyke Coelingh K. 1989. Neutralization epitopes of the F glycoprotein of respiratory syncytial virus: effect of mutation upon fusion function. $J$ Virol, 63:2941-50.

Beyer M, Bartz H, Horner K, et al. 2004. Sustained increases in numbers of pulmonary dendritic cells after respiratory syncytial virus infection. J Allergy Clin Immunol, 113:127-33.

Buckingham SC, Jafri HS, Bush AJ, et al. 2002. A randomized, doubleblind, placebo-controlled trial of dexamethasone in severe respiratory syncytial virus (RSV) infection: effects on RSV quantity and clinical outcome. J Infect Dis, 185:1222-8.

Carbonell-Estrany XLG, Hultquist M, Connor E; the Motavizumab Study Group. 2007. Phase III trial of motavizumab (MEDI-524), an enhanced potency respiratory syncytial virus (RSV)-specific monoclonal antibody (Mab) for the prevention of serious RSV disease in high-risk infants. Pediatric Academic Societies Annual Meeting; Toronto, Canada, 2007.

Chavez-Bueno S, Mejias A, Welliver RC. 2006. Respiratory syncytial virus bronchiolitis: current and future strategies for treatment and prophylaxis. Treat Respir Med, 5:483-94.

Chavez-Bueno S, Olsen K, Danton M, et al. 2005. Dynamics of respiratory syncytial virus (RSV) loads in hospitalized children. In: 45th Interscience Conference on Antimicrobial Agents and Chemotherapy; Washington, DC; 2005.

Cohen SA, Zanni R, Cohen A, et al. 2008. Palivizumab use in subjects with congenital heart disease: Results from the 2000-2004 Palivizumab Outcomes Registry. Pediatr Cardiol, 29:382-7.

Collins PL, Crowe. EJ. 2007. Respiratory syncytial virus and metapneumovirus. In: Knipe DM, ed. Fields Virology. Philadelphia: Lippincott Williams and Wilkins. pp 1601-46. 
DeVincenzo JP, Hall CB, Kimberlin DW, et al. 2004. Surveillance of clinical isolates of respiratory syncytial virus for palivizumab (Synagis)resistant mutants. J Infect Dis, 190:975-8.

Devincenzo JP. 2004. Natural infection of infants with respiratory syncytial virus subgroups A and B: a study of frequency, disease severity, and viral load. Pediatr Res, 56:914-7.

Fearns R, Collins PL. 1999. Role of the M2-1 transcription antitermination protein of respiratory syncytial virus in sequential transcription. J Virol, 73:5852-64.

Feltes TF, Cabalka AK, Meissner HC, et al. 2003. Palivizumab prophylaxis reduces hospitalization due to respiratory syncytial virus in young children with hemodynamically significant congenital heart disease. J Pediatr, 143:532-40.

Fonseca-Aten M, Michaels GM, Charsha-May MM, et al. 2007. Respiratory syncytial virus (RSV) prophylaxis among pediatric solid organ transplant (SOT) programs. 4th Congress of the IPTA On Pediatric Transplantation 2007; Cancun, Mexico.

Giebels K, Marcotte JE, Podoba J, et al. 2008. Prophylaxis against respiratory syncytial virus in young children with cystic fibrosis. Pediatr Pulmonol, 43:169-74.

Glezen WP, Taber LH, Frank AL, et al. 1986. Risk of primary infection and reinfection with respiratory syncytial virus. Am J Dis Child, 140:543-6.

Groothuis JR, Simoes EA, Levin MJ, et al. 1993. Prophylactic administration of respiratory syncytial virus immune globulin to high-risk infants and young children. The Respiratory Syncytial Virus Immune Globulin Study Group. N Engl J Med, 329:1524-30.

Hall CB, Douglas RG Jr, Geiman JM. 1976. Respiratory syncytial virus infections in infants: quantitation and duration of shedding. J Pediatr, 89:11-5.

Hall CB. 2000. Respiratory Syncytial Virus. Mandell: Principles and Practice of Infectious Diseases. 5 ed. Churchill Livingstone, Inc..

Hall WJ, Hall CB, Speers DM. 1978. Respiratory syncytial virus infection in adults: clinical, virologic, and serial pulmonary function studies. Ann Intern Med, 88:203-5.

Jafri HS, Chavez-Bueno S, Mejias A, et al. 2004. Respiratory syncytial virus induces pneumonia, cytokine response, airway obstruction, and chronic inflammatory infiltrates associated with long-term airway hyperresponsiveness in mice. J Infect Dis, 189:1856-65.

Johnson S, Oliver C, Prince GA, et al. 1997. Development of a humanized monoclonal antibody (MEDI-493) with potent in vitro and in vivo activity against respiratory syncytial virus. $J$ Infect Dis, 176:1215-24.

Johnson TR, Varga SM, Braciale TJ, et al. 2004. Vbeta14(+) T cells mediate the vaccine-enhanced disease induced by immunization with respiratory syncytial virus (RSV) G glycoprotein but not with formalin-inactivated RSV. J Virol, 78:8753-60.

Kuzik BA, Al-Qadhi SA, Kent S, et al. 2007. Nebulized hypertonic saline in the treatment of viral bronchiolitis in infants. J Pediatr, 151:266-70, 70 e1.

Malley R, DeVincenzo J, Ramilo O, et al. 1998. Reduction of respiratory syncytial virus (RSV) in tracheal aspirates in intubated infants by use of humanized monoclonal antibody to RSV F protein. $J$ Infect Dis, 178:1555-61.

Mejias A, Chavez-Bueno S, Rios AM, et al. 2004b. Anti-respiratory syncytial virus (RSV) neutralizing antibody decreases lung inflammation, airway obstruction, and airway hyperresponsiveness in a murine RSV model. Antimicrob Agents Chemother, 48:1811-22.

Mejias A, Chavez-Bueno S, Rios AM, et al. 2005b. Comparative effects of two neutralizing anti-respiratory syncytial virus (RSV) monoclonal antibodies in the RSV murine model: time versus potency. Antimicrob Agents Chemother, 49:4700-7.
Mejias A, Chavez-Bueno S, Sanchez P. 2005a. Respiratory syncytial virus prophylaxis. NeoReviews, e6(1):1-6.

Mejías A, Ríos C-BS, Fonseca-Aten AM, et al. 2004a. Comparative effect of an anti-respiratory syncytial virus monoclonal antibodies on viral replication and disease severity in the RSV murine model. In: 44th Interscience Conference on Antimicrobial Agents and Chemotherapy; 2004; Washington DC, MD.

Palivizumab (Synagis ${ }^{\circledR}$ ) package insert [online]. Accessed Sept 1, 2005. URL: http://www.medimmune.com/pdf/products/synagis_pi.pdf

Palivizumab, a humanized respiratory syncytial virus monoclonal antibody, reduces hospitalization from respiratory syncytial virus infection in high-risk infants. The IMpact-RSV Study Group. 1998; Pediatrics, 102:531-7.

Parnes C, Guillermin J, Habersang R, et al. 2003. Palivizumab prophylaxis of respiratory syncytial virus disease in 2000-2001: results from The Palivizumab Outcomes Registry. Pediatr Pulmonol, 35:484-9.

Psarras S, Papadopoulos NG, Johnston SL. 2004. Pathogenesis of respiratory syncytial virus bronchiolitis-related wheezing. Paediatr Respir Rev, 5(Suppl A):S179-84.

[PREVENT] Reduction of respiratory syncytial virus hospitalization among premature infants and infants with bronchopulmonary dysplasia using respiratory syncytial virus immune globulin prophylaxis. The PREVENT Study Group. 1997. Pediatrics, 99:93-9.

Red Book. 2006. Report of the Committee on Infectious Diseases. 27th ed. Elk Grove Village, IL: American Acedemy of Pediatrics.

Saez-Llorens X, Castano E, Null D, et al. 1998. Safety and pharmacokinetics of an intramuscular humanized monoclonal antibody to respiratory syncytial virus in premature infants and infants with bronchopulmonary dysplasia. The MEDI-493 Study Group. Pediatr Infect Dis $J$, 17:787-91.

Shay DK, Holman RC, Newman RD, et al. 1999. Bronchiolitis-associated hospitalizations among US children, 1980-1996. JAMA, 282:1440-6.

Sigurs N, Gustafsson PM, Bjarnason R, et al. 2005. Severe respiratory syncytial virus bronchiolitis in infancy and asthma and allergy at age 13. Am J Respir Crit Care Med, 171:137-41.

Simoes EA, Groothuis JR, Carbonell-Estrany X, et al. 2007. Palivizumab prophylaxis, respiratory syncytial virus, and subsequent recurrent wheezing. J Pediatr, 151:34-42, e1

Simoes EA, Sondheimer HM, Top FH Jr, et al. 1998. Respiratory syncytial virus immune globulin for prophylaxis against respiratory syncytial virus disease in infants and children with congenital heart disease. The Cardiac Study Group. J Pediatr, 133:492-9.

Spann KM, Tran KC, Chi B, et al. 2004. Suppression of the induction of alpha, beta, and lambda interferons by the NS1 and NS2 proteins of human respiratory syncytial virus in human epithelial cells and macrophages [corrected]. $J$ Virol, 78:4363-9.

Stein RT, Sherrill D, Morgan WJ, et al. 1999. Respiratory syncytial virus in early life and risk of wheeze and allergy by age 13 years. Lancet, 354:541-5.

Wang EE, Prober CG, Manson B, et al. 1984. Association of respiratory viral infections with pulmonary deterioration in patients with cystic fibrosis. $N$ Engl J Med, 311:1653-8.

Zhao X, Chen FP, Megaw AG, et al. 2004b. Variable resistance to palivizumab in cotton rats by respiratory syncytial virus mutants. J Infect Dis, 190:1941-6.

Zhao X, Chen FP, Sullender WM. 2004a. Respiratory syncytial virus escape mutant derived in vitro resists palivizumab prophylaxis in cotton rats Virology, 318:608-12. 
